\title{
弱可逆有限自动机的化合与分解
}

\author{
鲍 丰 \\ (中国科学院软件矿究所, 北京 100u80)
}

摘 要

本文证明了任何素数元延迟 1 步WIFA 可以分解出一个延迟元与一个无延迟 WIFA. 故而 $k$ 个素数元延迟 1 步WIFA 的化合可以分解为一个无延迟 WIFA 与 $k$ 个延讯元. 另外对于二元延迟 $\tau$ 步WIFA，上述分解可行的充要条件是其所有 状态的延迟步数均为 $\tau$.

\section{关键词 有限自动机、弱可逆、延迟、分解}

50 年代末 Huffman ${ }^{[1]}$ 首先提出了 “信息无损失有限状态机”的概念. 继而由 Even ${ }^{[2,3]}$ 将 “信息无损失”推广到五元组的有限自动机模型上. 从功能上讲, 作为序列转换器的有限自动 机“信息无损失”，实质上就是可逆. 最近十几年中,陶仁骥对有限自动机可逆性理论做了深入。 与系统的研究 ${ }^{[4-11]}$, 并提出了以有限自动机可逆性为基础的公开钥密码体制 ${ }^{[8]}$, 以及基于身份 的公开钥密码体制和数字签名 ${ }^{[1]}$. 目前, 有限自动机公开钥密码体制已得以实现 ${ }^{[12]}$, 并将投人 使用. 这进一步韭示出扸限自动机可逆性在密码学中的优势和潜力, 从而使对有限自动机可 逆性理论的更深入研究显得尤为重要.

文献 $[6,11]$ 中都提到了有限自动机化合的概念. 化合是从相对简单的自动机构造出结 构相对复杂一些的自刓机的一种手段. 化合保持有限自动机的可逆性, 具体来说, 一个延迟 $\tau_{1}$ 步 WIFA 与一个延迟 $\tau_{2}$ 步 WIFA 化合, 得到一个延迟 $\tau_{1}+\tau_{2}$ 步 WIFA. 但反过来, 一 个 WIFA 是否可以分解成两个或多个具有较其本身延迟步数更小的 WIFA 以及可以分解成 什么样的 WIFA 这样的分解问题, 对于非线性有限自动机来说是非常困难的问题. 由于对 非线性的情形缺少有力的数学工具, 非线性WIFA 的分解问题进展甚微, 目前有关这方面的 结果很少.

本文对素数元延迟 1 步 WIFA 得到了:

(1) 对自动机化合与分解理论的研究, 包括分析化合自动机的炶构及性质. 由素数元延 迟 1 步 WIFA 化合而得的 WIFA, 可以分解为一个延迟 0 步 WIFA 与一个 $\tau$ 步延迟元.

（2）一个延迟 0 步 WIFA 与一个 $\tau$ 步延迟元的化合必然是一个延迟 $\tau$ 步 WIFA, 且它 的所有状态的延迟步数均为 $\tau$. 本文证明了对于二元的情形, 它的反命题也是成立的. 对于 多元情形,这个问题有待进一步探讨. 


\section{1 基本摡念与记号}

一个有限自动机是一个五元组 $M=\langle X, Y, S, \delta, \lambda\rangle$, 其中 $X, Y, S$ 分别是输人、输出以 及状态字母表 (均为非空有限集); $\delta$ 是一个从 $S \times X$ 到 $S$ 的映射, $\lambda$ 是一个从 $S \times X$ 到 $Y$ 的映 射,分别称为下一状态函数与箱出函数.

称 $M=\langle X, Y, S, \delta, \lambda\rangle$ 是延造 $\tau$ 步䎊可逆的, 如果 $\forall s \in S, a, a^{\prime} \in X$ 以及 $\alpha, \alpha^{\prime} \in X^{\tau}$, 都 有 $\lambda(s, a \alpha)=\lambda\left(s, a^{\prime} \alpha^{\prime}\right)$ 䍀含 $a=a^{\prime}$.

称 $M=\langle X, Y, S, \delta, \lambda\rangle$ 是严格延迟 $\tau$ 步弱可逆的,如果 $M$ 是延迟 $\tau$ 步弱可逆但不是延迟 $x-1$ 步弱可逆.

如果 $M=\langle X, Y, S, \delta, \lambda\rangle$ 是一个萔可逆有限自动机（weakly invertible finite automa. ton, 简记 WIFA), 则 $\forall s \in S$, 必存在整数 $\tau \geqslant 0$, 使得对任何 $a, a^{\prime} \in X$ 及 $a, \alpha^{\prime} \in X^{\tau}$, 都 有 $\lambda(s, a \alpha)=\lambda\left(s, a^{\prime} \alpha^{\prime}\right)$ 推出 $a=a^{\prime}$. 称满足上述条件的最小的 $\tau$ 是 $s$ 的延迟步数.

显然， $M$ 是严格延迟 $\tau$ 步 WIFA 当且仅当 $M$ 是延迟 $\tau$ 步 WIFA 且 $M$ 至少有一个状态的 延迟步数为 $\tau$.

设 $M_{1}=\left\langle X, Y, S_{1}, \delta_{1}, \lambda_{1}\right\rangle, M_{2}=\left\langle Y, Z, S_{2}, \delta_{2}, \lambda_{2}\right\rangle$, 文献 [6] 中定义了 $M_{1}$ 与 $M_{2}$ 的化合 （自动机） $C\left(M_{1}, M_{2}\right)=\left\langle X, Z, S_{1} \times S_{2}, \delta, \lambda\right\rangle$, 其中

$$
\begin{aligned}
& \delta\left(\left\langle s_{1}, s_{2}\right\rangle, x\right)=\left\langle\delta_{1}\left(s_{1}, x\right), \delta_{2}\left(s_{2}, \lambda_{1}\left(s_{1}, x\right)\right)\right\rangle, \\
& \lambda\left(\left\langle s_{1}, s_{2}\right\rangle, x\right)=\lambda_{2}\left(s_{2}, \lambda_{1}\left(s_{1}, x\right)\right), \quad\left\langle s_{1}, s_{2}\right\rangle \in S_{1} \times s_{2}, x \in X .
\end{aligned}
$$

从功能上看 $M_{1}$ 与 $M_{2}$ 的化合相当于将 $M_{1}$ 与 $M_{2}$ 串联使用.

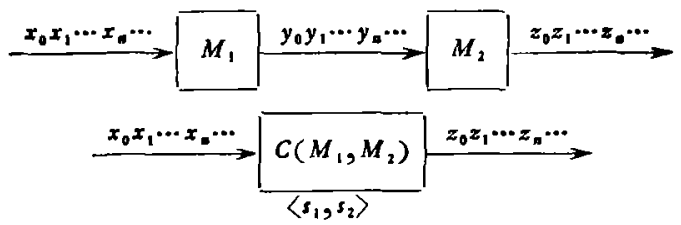

显然，如果 $M_{1}$ 是延迟 $\tau_{1}$ 步 WIFA, $M_{2}$ 是延迟 $\tau_{2}$ 步 WIFA，则 $C\left(M_{1}, M_{2}\right)$ 是延迟 $\tau_{1}+\tau_{2}$ 步 WIFA.

为了更方便地研究自动机的化合，如不作特别声明，以下我们讨论的有限自动机均有相同 的输入输出字母表. 称 $M=\langle X, X, S, \delta, \lambda\rangle$ 为 $X$ 上的有限自动机; 若 $|X|=n$, 则称 $M$ 是 $n$ 元有限自动机.

设 $M_{1}$ 与 $M_{2}$ 都是 $X$ 上有限自动机, 则 $C\left(M_{1}, M_{2}\right)$ 仍是X上有限自动机. 这样化合就松 成了字母表 $X$ 上的有限自动机间的一个运算. 我们以后记 $C\left(M_{1}, M_{2}\right)$ 为 $M_{1} \cdot M_{2}$.

不难证明化合在同构意义下满足结合律, 即 $\left(M_{1} \cdot M_{2}\right) \cdot M_{3}$ 同构于 $M_{1} \cdot\left(M_{2} \cdot M_{3}\right)$, 因此可记 $M_{1}, M_{2}, M_{3}$ 的化合为 $M_{1} \cdot M_{2} \cdot M_{3}$, 或更简单地, 记为 $M_{1} M_{2} M_{3}$.

问理, 对 $X$ 上任意 $k$ 个有限自刓机 $M_{1}, M_{2}, \cdots, M_{k}$, 记它们的化含为 $M_{1} M_{2} \cdots M_{k}$, 记 其状态为 $\left\langle s_{1}, s_{2}, \cdots, s_{k}\right\rangle$, 其中 $s_{i}$ 是 $M_{i}$ 的一个状态， $i=1,2, \cdots, k$.

设 $M_{1}, M_{2}, \cdots, M_{k}, M_{1}^{\prime}, M_{2}^{\prime}, \cdots, M_{k}^{\prime}$ 是 $X$ 上有限自动机, 不难证明如下结果:

定理 1 (1) 如喿 $s_{i}$ 是 $M_{i}$ 的状态, $s_{i}^{\prime}$ 是 $M_{i}^{\prime}$ 的状态, 且 $s_{i} \sim s_{i}^{\prime}, i=1,2, \cdots, k$, 那么 对化合自动机 $M . M_{2} \cdots M_{k}$ 与 $M_{1}^{\prime} M_{2}^{\prime} \cdots M_{k}^{\prime}$ 有 


$$
\left\langle s_{1}, s_{2}, \cdots, s_{k}\right\rangle \sim\left\langle s_{1}^{\prime}, s_{2}^{\prime}, \cdots, s_{k}^{\prime}\right\rangle \text {; }
$$

(2) 如果 $M_{i}<M_{i}^{\prime}, i=1,2, \cdots, k$, 则有

$$
M_{1} M_{2} \cdots M_{k}<M_{1}^{\prime} M_{2}^{\prime} \cdots M_{k}^{\prime} \text {; }
$$

(3) 如果 $M_{i} \sim M_{i}^{\prime}, i=1,2, \cdots, k$, 则有

$$
M_{1} M_{2} \cdots M_{k} \sim M_{1}^{\prime} M^{\prime} \cdots M_{k}^{\prime} \text {. }
$$

这里～表示状态之间或自动机之间的等价， $M_{1}<M_{2}$ 表示自动机 $M_{1}$ 弱于 $M_{2}$ 円.

\section{2 延迟 1 步 WIFA}

如果 $M_{1}, M_{2}, \cdots, M_{k}$ 是 $X$ 上的 $k$ 个延迟 0 步 WIFA, 则 $M_{1} M_{2} \cdots M_{k}$ 也是延迟 0 步 WIFA. 因此对 WIFA 化合与分解性质的研究, 就要从最基本的延迟 1 步WIFA 开始.

下面给出几个记号. 设 $M-\langle X, Y, s, \delta, \lambda\rangle$ 是有限自动机, $s \in S, y \in Y$, 定义

$\operatorname{Out}(s)=\{y \in Y$ ! 存在 $x \in X$, 使得 $y-\lambda(s, x)\}$,

$d(s)=\mid$ Out $(s) \mid$,

$\ln (s, y)-\{x \in X \mid \lambda(s, x)-y\}$,

$S_{\imath}(s)-\left\{\imath \in S \mid\right.$ 存在 $\left.a \in X^{*}, \imath-\delta(s, a)\right\}$,

$S_{1}(s, y)=\{t \in S \mid$ 存在 $x \in X$, 使得 $\imath-s(s, x)$ 且 $\lambda(s, x)-y\}$.

定理 $2 M$ 是 $X$ 上延迟 1 步弱可逆有限自动机, 如果 $M$ 的某一状态 $s$ 满足对任何 $M$ 中状 都有 $d(s) \leqslant d(t)$, 则有

(1) $d(s)$ 是 $|X|$ 的因数, 即 $d(s)|| X \mid$;

(2) $\forall t \in S_{t}(s), d(\imath)-d(s)$;

(3) $\forall x \in \operatorname{Out}(s),|\operatorname{In}(s, x)|-\frac{|X|}{d(s)}$;

(4) 如 $x \in \operatorname{Out}(s)$, 则对所有的 $\imath \in S,(s, x), \operatorname{Out}(t)$ 互不相交, 且它们的并为 $X$.

证它文献 [10] 中定理 8 .

下面我们对有限自动机加上更强的限制, 以去掉一些“多余”的状态而只保留自动机的核 心部分.

定义 称有限自动机 $M-\langle X, Y, S, \delta, \lambda\rangle$ 是强连通的, 如果 对任何 $s, t \in \delta$, 存在 $a \in X^{*}$, 使得 $s-\delta(t, \alpha)$.

任何有限自动机都有强连通子自动机

定理 3 任何 $n$ 元强连通严格延迟 1 步的 WIFA 其所有状态的延迟步数均为 1 .

证 设 $M=\langle X, X, s, d, \lambda\rangle$ 是严格延迟 1 步 WIFA, $|X|=n$. 任何 $s \in S, s$ 的延迟 步数 $\leqslant 1$. 又设 $t \in S$ 且 $d(t)$ 最小, 则 $d(t)<n$, 否则 $M$ 不是严格延迟 1 步的. $M$ 是强连通 的, 所以 $s-S_{i}(t)$, 由定理 2 知对任何 $s \in S$, 都有 $d(s)-d(t)<n$, 故 $s$ 的延迟步数为 I.

定理 $4 M-\langle X, X, S, \delta, \lambda\rangle$ 是素数元强连通的严格延迟 1 步 WIFA, 则对任何 $s \in S$ 有 $d(s)=1$. 设 $x_{1} x_{2} \cdots x_{*}$ 是 $X$ 的一个排列, 则对任何 $s \in S$, Out $\left(\delta\left(s, x_{1}\right)\right), \cdots, \operatorname{Out}(\delta(s$, $\left.\boldsymbol{x}_{\mathrm{s}}\right)$ ）亦构成 $X$ 的一个排列.

证 综合定理 2 与定理 3. 
下面我们来考虑 $X$ 上的一个特殊延迟 1 步 WIFA. $M_{d}=\langle X, X, X, \delta, \lambda\rangle$, 其状态集与 输入输出集相同，对任何 $x, y \in X$,

$$
\left\{\begin{array}{l}
\delta(x, y)=y, \\
\lambda(x, y)=x .
\end{array}\right.
$$

$M_{d}$ 的逻辑网络表达就是 $X$ 上的一个记忆元件:

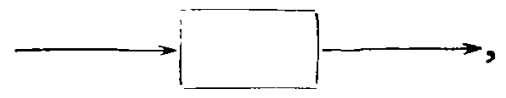

$\forall x_{0}, x_{1}, x_{2}, \cdots, x_{n} \in X$, 有

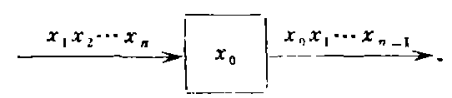

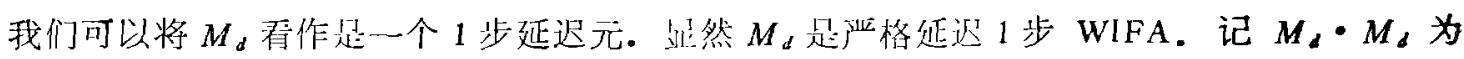
$M_{2 d}$; 同理,将 $\tau$ 个 $M_{d}$ 的化合记为 $M_{\tau d}$, 显然 $M_{\tau d}$ 的迬辑网络实现为

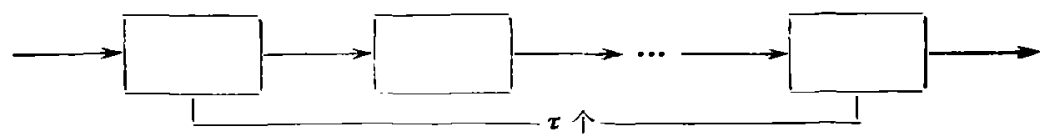

$M_{\tau d}$ 是严格延迟 $\tau$ 步 WIFA.

定理 $5 M$ 是 $X$ 上有限自动机, $M_{d}$ 是 $X$ 上延迟元, 则 $M_{d} M<M M_{d}$.

证 设 $M=\langle X, X, s, \delta, \lambda\rangle$, 对任何 $s \in S, x_{0} \in X,\left\langle x_{0}, s\right\rangle$ 是 $M_{d} M$ 的一个状态. 我 们考虑 $M M_{d}$ 的状态 $\left\langle\delta\left(s, x_{0}\right), \lambda\left(s, x_{1}\right)\right\rangle$. 对任何 $x_{1}, x_{2}, \cdots, x_{n} \in X$, 令 $\lambda\left(s, x_{0} x_{1} \cdots x_{n}\right)=$ $y_{0} y_{1} \cdots y_{n}, M_{d} M=\left\langle X, X, X \times S, \delta_{1}, \lambda_{1}\right\rangle, M M_{d}=\left\langle X, X, s \times X, \delta_{2}, \lambda_{2}\right\rangle$, 则有

$$
\begin{aligned}
& \lambda_{1}\left(\left\langle x_{0}, s\right\rangle, x_{1} x_{2} \cdots x_{n}\right)-y_{0} y_{1} \cdots y_{n-1}, \\
& \lambda_{2}\left(\left\langle\delta\left(s, x_{0}\right), y_{0}\right\rangle, x_{1} x_{2} \cdots x_{n}\right)=y_{0} y_{1} \cdots y_{n-1} .
\end{aligned}
$$

由 $x_{i}, i-1,2, \cdots, n$ 的任意性知

$$
\left\langle x_{0}, s\right\rangle \sim\left\langle\delta\left(s, x_{0}\right), \lambda\left(s, x_{0}\right)\right\rangle .
$$

所以 $M_{d} M<M M_{d}$.

由定理 5 可以春出对任何正整数 $\tau$, 也们 $M_{\tau d} M<M M_{\tau d}$ 。

定理 $6 M$ 是素数元强连通严格延迟 1 步 WIFA, 则必存在延迟 0 步WIFA $M_{0}$, 使得

$$
M<M_{0} M_{d} \text {. }
$$

证 $M=\langle X, X, s, \delta, \lambda\rangle,|X|$ 是素数. 由定理 4, 对任何 $s \in S, d(s)-1$, 即 $\forall x$, $x^{\prime} \in X, \lambda(s, x)-\lambda\left(s, x^{\prime}\right)$. 记 $\lambda(s, x)$ 为 $\lambda(s)$. 沟造有限自动机 $M_{0}-\left\langle X, X, s, \delta_{0}, \lambda_{0}\right\rangle$, 定 义

$$
\left\{\begin{array}{l}
\delta_{0}(s, x)-\delta(s, x), \\
\lambda_{0}(s, x)-\lambda(\delta(s, x)) .
\end{array}\right.
$$

由定理 4 知 $\forall s \in S . \lambda_{0}(s, x)$ 构成 $X$ 上的 1-1 映射, 因此 $M_{0}$ 是延迟 0 步 WIFA. $M$ 的任一状 态 $s$ 等价于 $M_{0} M_{d}$ 的状态 $\langle s, \lambda(s)\rangle$, 这可由 $\lambda_{0}$ 的定义看出. 因此有 $M<M_{0} M_{d}$.

\section{3 延迟 $\tau$ 步 WIFA 的分解}

对于一般的非线性延迟 $\tau$ 步 WIFA，分解问题远远没有解决，本节给出两种特殊情况下 
的分解性质.

定理 $7 M_{1}, M_{2}, \cdots, M_{\mathrm{r}}$ 均为 $X$ 上强连通的严格延迟 1 步 WIFA, 且 $|X|$ 是素数, 则其 化合 $M_{1} M_{2} \cdots M_{\tau}$ 是严格延迟 $\tau$ 步 WIFA, 并且存在延迟 0 步 WIFA $M_{0}$, 使得 $M_{1} M_{2} \cdots$ $M_{\tau}<M_{0} M_{s d}$.

证 由定理 6, 存在延迟 0 步 WIFA $M_{0 i}$, 使得 $M_{i}<M_{s_{b}} M_{d}, t=1,2, \cdots, \tau$. 因此

$$
\begin{aligned}
M_{1} M_{2} \cdots M_{\mathrm{r}} & <M_{01} M_{d} M_{\mathrm{J} 2} M_{d} \cdots M_{3 \tau} M_{d} \\
& <M_{01} M_{02} \cdots M_{\mathrm{dr}} M_{d} \cdots M_{d}=M_{\lrcorner} M_{r d},
\end{aligned}
$$

其中 $M_{0}-M_{01} M_{02} \cdots M_{0 \tau}$ 是延迟 0 步 WIFA, $M_{\tau d}$ 是 $\tau$ 个 $M_{d}$ 的化合.

上述定理说明了由 $\tau$ 个素数元强连通严格延迟 1 步 WIFA 化合得到的有限自动机, 必 是严格延迟 $\tau$ 步的, 且每个状态的延迟步数均为 $\tau$.

对于严格延迟 $\tau$ 步且每一个状态们延迟步数均为 $\tau$ 的 WIFA 是否一定可以你解出延迟 元尚不清楚. 但对二元的情玨我们有如下结果.

定理 $8 M$ 是二元延迟 $\tau$ 步 WIFA， $M$ 的所有状态的延迟步数均为 $\tau$ 的充分必要条件是 存在延迟 0 步 WIFA $M_{0}$, 使得 $M<M_{0} M_{s d}$.

为了证明定理 8 , 我们先考虑下䤄两个引理.

引理 $1 M=\langle X, X, S, \delta, \lambda\rangle$ 是二元延迟 $\tau$ 步 WIFA. $M$ 的所有状态的延迟步数均为 $\tau, \tau>0$ 的充分必要条件是 $\forall s \in S, k<\tau$,

$$
\left|\bigcup_{s \in t(s)} \operatorname{Our}(t)\right|=1
$$

且 $\forall x_{0}, x_{1} \in X, x_{0} \neq x_{1}$ 有

$$
\bigcup_{i \in s_{\tau-1}\left(\delta\left(s, x_{0}\right)\right)} \operatorname{Out}(t) \neq \bigcup_{t \in s_{\tau}-1^{\left(\delta\left(s, x_{1}\right)\right)}} \operatorname{Out}(t),
$$

其中 $S_{k}(s)=\left\{t \in S \mid\right.$ 存在 $a \in X^{k}$ 使 $\left.t-\delta(s, a)\right\}$.

证 充分性显然. 下面证明必要性.

由于 $M$ 的每个状态延迟步均为 $\tau, \tau>0$, 因此 $\forall s \in S, d(s)=1$. 故可记 $\lambda(s, x)$ 为 $\lambda(s)$. 这样, 对 $k=0,(*)$ 式成立.

假设对 $k=r<\tau-1$ ，（*)式成立. 我们证明对 $k=r+1 ，(*)$ 式亦成立. 不妨设 $X-\left\{x_{0}, x_{1}\right\}$, 对任何 $s \in S$, 由假设知

$$
\begin{aligned}
& \left|\bigcup_{s \in S_{r}\left(\delta\left(s, x_{\theta}\right)\right)} \operatorname{Out}(\boldsymbol{t})\right|-1, \\
& \left|\bigcup_{1} \bigcup_{S p(s(t, x))} \operatorname{Out}(t)\right|=1 \text {. }
\end{aligned}
$$

由于s的延迟步数为 $\tau$, 因此必有

$$
\bigcup_{i \varepsilon S_{r}\left(\delta\left(s, x_{0}\right)\right)} \operatorname{Out}(t)=\bigcup_{t \in S_{r}(\delta(s, s, 1))} \operatorname{Out}(t),
$$

否则 $s$ 的延迟步数为 $r+1$, 但 $r+1<\tau$.

而 


$$
\bigcup_{i \in S_{r+1}(s)} \operatorname{Out}(t)-\left[\bigcup_{r \in S_{r}\left(\delta\left(s, x_{0}\right)\right)} \operatorname{Out}(t)\right] \cup\left[\bigcup_{r \in S_{r}\left(\delta\left(s, x_{1}\right)\right)} \operatorname{Out}(t)\right]
$$

因此

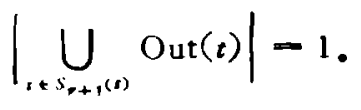

由数学归纳法知, $(*)$ 式得证.

(**) 式成立是显然的, 因为否则的话, $s$ 的延迟步数大于 $\tau$.

引理 $2 M$ 是二元延迟 $\tau$ 步 WIFA, $\tau>0$, 且每个状态的延迟步数均为 $\tau$, 则存在延迟 $\tau \rightarrow 1$ 步 WIFA $M^{\prime}, M^{\prime}$ 的每个状态的延迟步数均为 $\tau-1$, 使得 $M<M^{\prime} M_{d}$.

证 设 $M=\langle X, X, S, \delta, \lambda\rangle, X-\left\{x_{0}, x_{1}\right\}$. 对 $\tau=1$ 的情形,定理 6 已给出证明. 下面 考虑 $t>1$ 约情形。

构造 $M^{\prime}=\left\langle X, X, S^{\prime}, \delta^{\prime}, \lambda^{\prime}\right\rangle$, 其中 $S^{\prime}-S$. 令

$$
\left\{\begin{array}{l}
\delta^{\prime}(s, x)-\delta(s, x), \\
\lambda^{\prime}(s, x)-\lambda(\delta(s, x)),
\end{array}\right.
$$

这里由于 $\delta(s, x) \in S$, 故 $|\operatorname{Out}(\delta(s, x))|-1$, 记 $\delta(s, x)$ 的输出为 $\lambda(\delta(s, x))$.

从 $M^{\prime}$ 的构造不难看出, 对任何 $s \in S, M$ 的状态 $s$ 等价于 $M^{\prime} M$, 的状态 $\langle s, \lambda(s)\rangle$. 因 乩就有 $M<M^{\prime} M_{d}$ 。 下面证明 $M^{\prime}$ 是延迟 $\tau-1$ 步 WIFA 且 $M^{\prime}$ 的每一个状态的延迟步数 的为 $\tau-1$.

由引理 $1, \forall s \in S, k<\tau$ 有

$$
\left|\bigcup_{1: s_{k}(s)} \operatorname{Out}(t)\right|-1 \text { 及 } \bigcup_{i \in s_{z-1}\left(s\left(s, s, s_{0}\right)\right)} \operatorname{Out}(t) \neq \bigcup_{i \in s_{z-1}\left(s\left(s, s_{1}\right)\right)} \operatorname{Out}(t) \text {. }
$$

从 $M^{\prime}$ 的构造 (主要是 $\lambda^{\prime}$ 的构造) 有, $\forall s \in S-s^{\prime}$,

$$
\begin{aligned}
& \left.\bigcup_{s \in s_{k}(s)} \operatorname{Out}(t)\right|_{\text {相对于M }}-\left\{x \in X \mid \text { 存在 } x^{\prime} \in X, a \in X^{k} \text {, 使 } x-\lambda\left(\delta(s, x), x^{\prime}\right)\right\}, \\
& \left.\bigcup_{t \in s_{k+1}(s)} \operatorname{Out}(t)\right|_{\text {相对于 } M^{\prime}}-\left\{x \in X \mid \text { 存在 } x^{\prime} \in X, a \in X^{k+1} \text { 使 } x-\lambda^{\prime}\left(\delta(s, a), x^{\prime}\right)\right\} .
\end{aligned}
$$

不难看出上两式的右端相等. 因此对 $M^{\prime}$ 的任何状态 $s \in S^{\prime}, k<\tau-1$ 有

$$
\left|\bigcup_{s \in S_{k}(s)} \operatorname{Out}(t)\right|-1 \text { 以及 } \bigcup_{r \in s_{\tau-2}\left(\delta\left(s, x_{0}\right)\right)} \operatorname{Out}(t) \neq \bigcup_{t \in s_{\tau-1}\left(\delta\left(s, s_{1}\right)\right)} \operatorname{Out}(t) \text {. }
$$

由引理 1 知 $M^{\prime}$ 是延迟 $\tau-1$ 步 WIFA 且 $M^{\prime}$ 的每一个状态的延迟步数为 $\tau-1$.

定理 8 的证 充分性显然. 必要性的证明只要重复使用引理 2 即可.

\section{参考文献}

[1] Huffman, D. A., IRE Trans. on Cireuir Theory, May, Special Supplement, 1959, 6: $41-59$.

[2] Even, S., Swirching Circwit Theory and Logic Design, 1962, 144-147.

[3] Even, S., IEEE Trans. on Electronic Computers, 1965, EC-14(4): $561-569$.

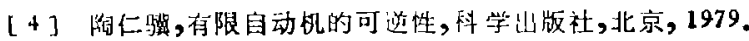

[5]陶仁槕,科学通报，1982,27(7): 406-408. 
[6] Too Renji, Chen Shihua, J. of Compuier Science and Technology, 1986, 1(1): 9-18.

[7] 陶仁嚁,自动机引论,科学出版社,北京, 1986.

(8) Tao Reaji, ICAL.P' 88, Ausomata. Languages and Programming, Springer-Verlag, Berlin, 1988, 489501.

19] Zhu Xinjie, $f$ of Computer Science and Technology, 1989, 4(2); 163-171.

[10] Bao Feng, Advances in Chinese Computer Science, World Scientific, Singapore, 1991. 3: $1-24$.

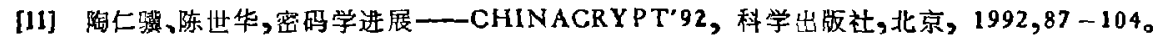

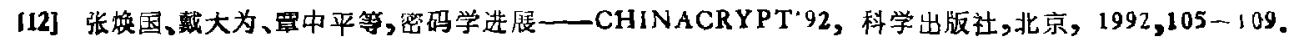

